\title{
Real Time Rasperi Pi Facial Emotion Recognition using Pca Improved
}

\author{
Burgoji Santhosh Kumar, Sudhir Kumar Sharma, K Haripal Reddy
}

\begin{abstract}
Emotions are first-rate way of communicating information and on occasion it deliver extra records than words. These days, there has been large hobby in automatic popularity of human emotion because of its huge spread utility in protection, surveillance, advertising, commercial, and human -laptop interaction. To speak with a laptop in a natural way, it will likely be applicable to apply more herbal modes of human conversation primarily based on voice, gestures and facial expressions. In this paper, a holistic method to facial expression recognition is to propose which captures the variant in facial functions in temporal domain and classifies the series of images in extraordinary feelings. The dimensionally of the Eigen area is reduced the use of fundamental aspect evaluation (PCA). Through projecting the following face photographs into primary Eigen guidelines, the variation pattern of the acquired weight vector is modeled to categories it into exclusive feelings. As a result of the versions of expressions for exceptional humans and its intensity, someone particular approach for emotion popularity is followed. The use of the gray scale pix of the frontal face, the machine is able to classify 4 simple emotions which include happiness, disappointment, marvel, and disgust.
\end{abstract}

Keywords: Real time embedded design, facial face recognition, Dimensionability Reduction, Human computer Interface.

\section{INTRODUCTION}

As one of the maximum a success packages of picture evaluation of understanding, face popularity was lately received great attention, especially for the course of the beyond several years. Face has gained sizeable significance in figuring out the state and expressions of a Human. Race, sex, age, and emotions are complex set of objects with so many features that can range all of the time based totally at the affect of external surroundings. Due to technological improvements, there may be an arousal of the world in which individual and shrewd robots stay together. recognition the emotional kingdom of someone by reading him/her facial features seem challenging but additionally important commonly because of the truth that most of the times facial expressions aren't most effective the direct emotional outcome but additionally result from the extraction of the that means of standards.

\section{LITERATURE SURVEY}

Highlighting inside the area of digital image Processing, the Human facial features reputation is one of the maximum active study place inside the discipline of Human computer interplay (HCI), clever Environments, scientific applications, synthetic wise based are totally robotics and automatic access manipulate. Spotting facial expressions are complicated for undertaking to complete a numerous obstacles which exist in lightning situation. Basic six face expressions are glad, wonder, Disgust, unhappy, angry, worry. As according to Meharabian [2], fifty five expressions became a major modality. As an example, clever devices like laptops or robots can sense/apprehend the human's intension from their expression on going to the beneficial machine to assist them by using giving suggestions or proposals as in keeping with their need. Improvements of Automatic facial expression and facial AU (movement unit) have attracted a great deal interest for the upcoming years because of its potential applications. In this paper, introducing the international function techniques which might be broadly used to detect in addition to perceive the human facial features from virtual snap shots for given dataset if any. Face Expression techniques can be divided into 3 predominant steps so that the face in an image is understood for further processing. Facial characteristic extraction is the approach used to symbolize the facial expressions and subsequently category step by step which classifies the capabilities extracted in the precise expressions.

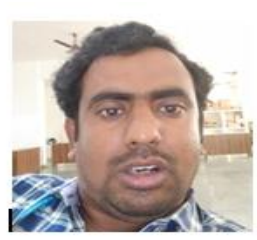

Fear

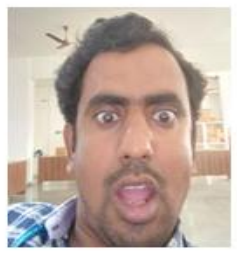

Surprise

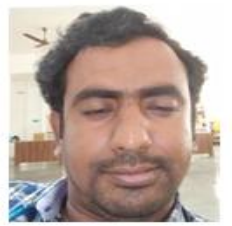

Sad

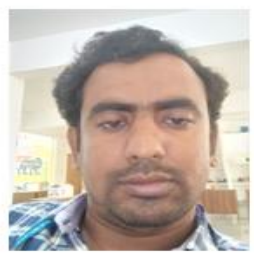

Happy

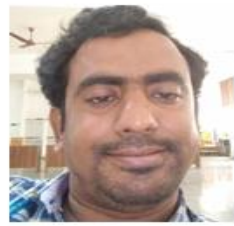

Disgust
Figure 2: Six Basic Human Expressions

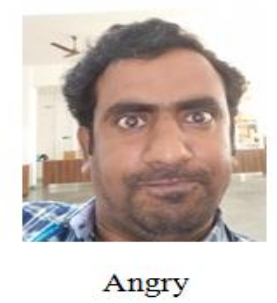

Revised Version Manuscript Received on July 18, 2019.

Burgoji Santhosh Kumar, Assistant Professor, Anurag Group of Institutions, Hyderabad, Telangana, India.

Prof. (Dr).Sudhir Kumar Sharma, Joint Director \& HOD ECE, Jaipur National University, Rajasthan, Jaipur India.

K Haripal Reddy, Associate Professor, Anurag Group of Institutions, Hyderabad, Telangana, India. 


\section{PROPOSED METHOD}

Recognization of Human facial expression to diverse feature strategies is given attention. Some of the methods were evolved for extracting features to face photos are primary element evaluation (PCA), Linear Discriminate analysis (LDA), Gabor filter out/energy, Line aspect Mapping (LEM), Help Vector system, Active look model (AAM) and the use of SIFT descriptor.

Facial Expression recognition can be categorized in to two major approaches.

i) Look primarily and ii) version based popularity techniques. To get feature of eyebrows, eyes and mouth rough contour estimation ordinary (RCER) is used. To enhance the precision of eye and mouth point contour detection technique (PCDM)[sixteen] is used. They use action devices (AU) for describing the fundamental actions of face muscle mass. They defined 30 facial traits points of eye, mouth and eyebrow for facial expression by using AU

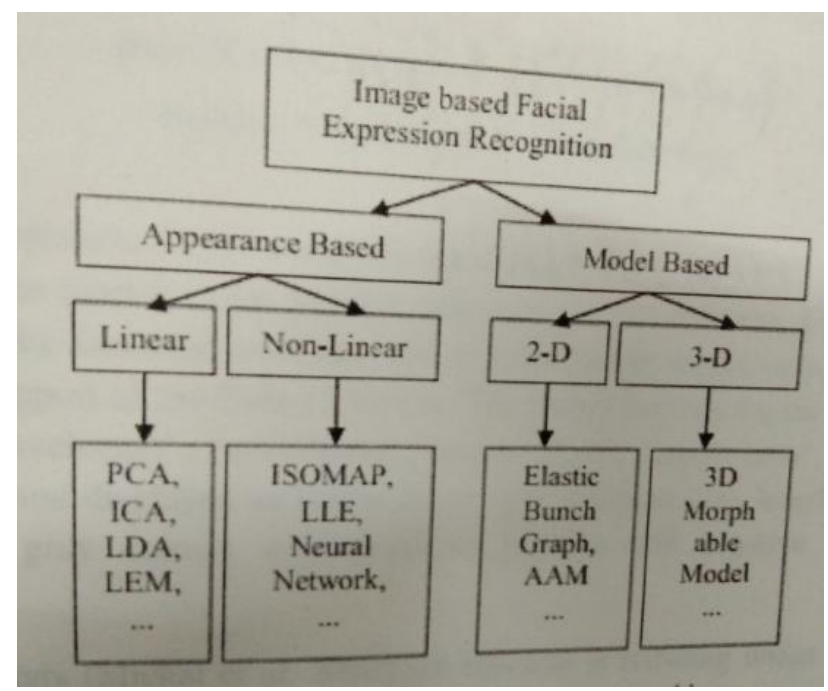

Figure 3. Category of Facial expression Recognition.

\section{RESULTS}

The implementation part has been done in MATLAB environment. The implementation results of the proposed technique can be shown in below figure.

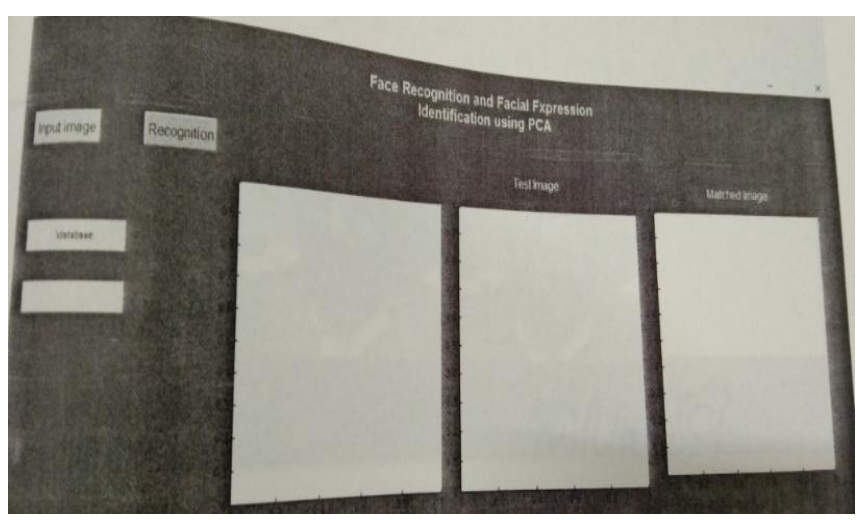

Figure 4. This is how window looks as soon as we run the program.

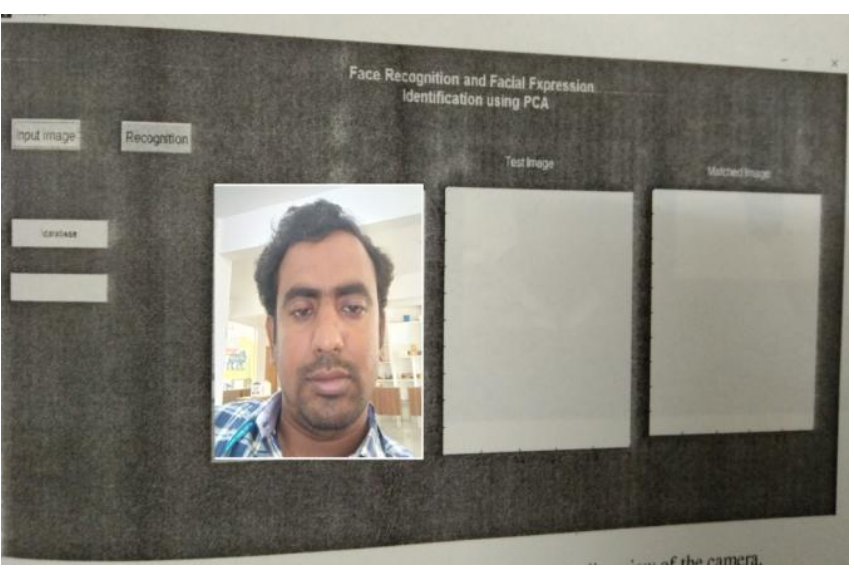

Figure 5. The camera starts and the program runs the live of the camera

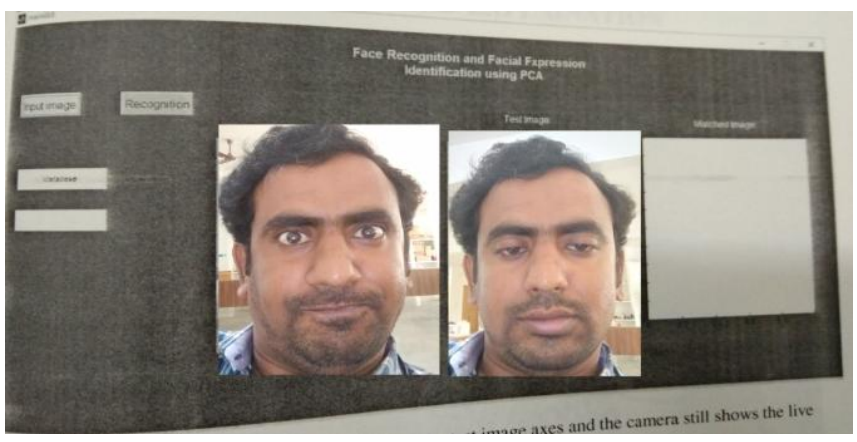

Figure 6. The captured image is shown in the test image axes and the camera still shows the live view of the camera in the first axes.

\section{Accuracy Table:}

\begin{tabular}{|l|l|}
\hline Facial Expressions & Accuracy \\
\hline Happy & 98.15 \\
\hline Sad & 95.62 \\
\hline Surprise & 99.19 \\
\hline Neutral & 98.62 \\
\hline
\end{tabular}

\section{CONCLUSION}

The facial popularity system may supply for research paintings which contributes to a resilient face recognition version based at the mapping of behavioral traits by the physiological biometric traits. From the physiological characteristics of human face to numerous expressions which may includes happiness, unhappiness, worry, anger, marvel and disgust these are related to geometrical systems which restored as base matching template for the popularity machine

\section{REFERENCES}

1. Black, M. J. and Yacoob, Y. Tracking and recognizing rigid and non-rigid facial motions using local parametric model of image motion. In Proceedings of the International Conference on Computer Vision, pages

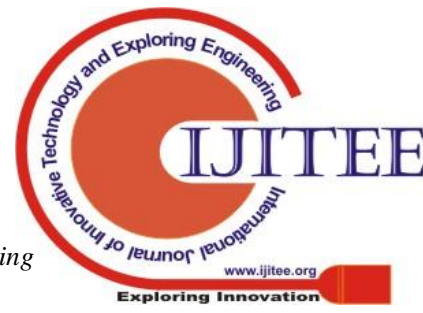


374-381. IEEE Computer Society, Cambridge, MA, 1995.

2. Boersma, P., Weenink, D., Praat Speech Processing Software, Institute of Phonetics Sciences of the University of Amsterdam.

3. Burges, C. A tutorial on support vector machines for pattern recognition. Dat Mining and Know. Disc., vol. 2(2), pp. 1-47, 1998.

4. Chen, L.S., Huang, T. S., Miyasato T., and Nakatsu R. Multimodal human emotion / expression recognition, in Proc. of Int. Conf. on Automatic Face and Gesture Recognition, (Nara, Japan), IEEE Computer Soc., April 1998

5. Chen, L.S., Huang, T.S. Emotional expressions in audiovisual human computer interaction. Multimedia and Expo, 2000. ICME 2000. 2000 IEEE International Conference on, Volume: 1, 30 July-2 Aug. 2000. Pages: 423 - 426 vol. 1

6. A. De, A. Saha, and M. C. Pal, "A Human Facial Expression Recognition Model Based on Eigen Face Approach," Procedia Comput. Sci., vol. 45, pp. 282-289, 2015.

7. A. Mehrabian, "Albert Mehrabian Communication Studies," $2013 . \quad$ [Online]. Available: http://www.iojt-dc2013.org/ /media/Microsites/Files/IOJ T/11042013-Albert-Mehrabian-Communication-Studies. ashx. [Accessed: 24-Jun-2015].

8. B. Kurt, V. V Nabiyev, and Y. Bekiroglu, "Yüz İfadelerinin Tanınması,” [Accessed: 01-Jun-2015] 